\title{
Constraining the Dark Sector with the Mono-jet signature with the ATLAS detector at the LHC
}

\author{
Elena Pompa Pacchi ${ }^{a, b, *}$, on behalf of the ATLAS Collaboration \\ ${ }^{a}$ Sapienza Università di Roma, Piazzale Aldo Moro 5, Roma, Italia \\ ${ }^{b}$ Istituto Nazionale di Fisica Nucleare sezione di Roma \\ E-mail: elena.pompa.pacchi@cern.ch
}

This work presents the reinterpretation of the search for final states with at least one energetic jet and missing transverse momentum in the context of dark sector models with long-lived neutral particles. Results are based on $139 \mathrm{fb}^{-1}$ of data collected in $p p$ collisions at $\sqrt{s}=13 \mathrm{TeV}$ by the ATLAS experiment during Run-2 at LHC. Decays of the Standard Model Higgs boson and of a new neutral heavy scalar mediator into long-lived particles are investigated in different channels. The resulting constraints generally extend those from dedicated searches at large proper lifetimes.

The Ninth Annual Conference on Large Hadron Collider Physics - LHCP2021

7-12 June 2021

Online

${ }^{*}$ Speaker 


\section{Motivations and signal models}

Final states with large missing transverse momentum $E_{\mathrm{T}}^{\text {miss }}$ recoiling against at least one energetic jet (monojets) provide a unique probe to search for processes from physics beyond the Strandard Model (SM) in which non-interacting particle are produced. The monojet search [1] based on the dataset collected by the ATLAS experiment [2] during Run-2 at the Large Hadron Collider (LHC) [3] is in this study [4] reinterpreted in terms of Dark Sector models in which the Higgs boson acts as portal to new physics and where the existence of Long Lived Particles (LLPs) is foreseen. Two signal processes are considered: one predicted in the Falkowski-Ruderman-Volansky-Zupan (FRVZ) model [5, 6] (Figure 1(a)), where the Higgs boson decays into a pair of dark fermions $f_{d}$, and one, in Figure 1(b), with the Higgs boson decaying into a pair of dark scalars $s$.

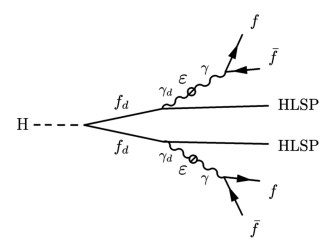

(a)

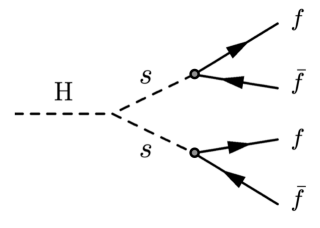

(b)

Figure 1: (a) Higgs decay predicted by the FRVZ model, with the blobs representing the mixing, governed by $\varepsilon$, of $\gamma_{d}$ with $\gamma, f_{d}$ the intermediate states coupled to the Higgs and HLSP the undetected Hidden Lightest Stable Particle. (b) Higgs decay into two scalar particles $s$.

The first process results into cascade decays into HLSPs, which go undetected and produce $E_{\mathrm{T}}^{\text {miss }}$, and dark photons, $\gamma_{d}$. The $\gamma_{d}$ is massive (with $m_{\gamma_{d}} \in[0.2,2] \mathrm{GeV}$ ) and mixes with the SM $\gamma$ via an interaction governed by the parameter $\varepsilon$. The smaller is $\varepsilon$, the larger is the proper lifetime of the $\gamma_{d}, \tau_{d}$. The off-shell $\gamma$ then decays into a pair of light SM fermions too. A similar signature is obtained for the second model, where the $s$ scalar decays, with proper lifetime $\tau_{s}$, into pairs of light SM fermions too. Both these models have been investigated by dedicated searches $[7,8,9,10,11]$ optimized for different $c \tau$ regimes, looking for either prompt or displaced decays of the $\gamma_{d}$ or of the $s$ scalar. The scenario considered in this analysis investigates the possibility that the system is boosted by Initial State Radiation (ISR) and $c \tau_{d}\left(c \tau_{s}\right)$ is so large that the $\gamma_{d}(s)$ decays outside the detector, yielding $E_{\mathrm{T}}^{\text {miss }}$, leading to a monojet signature. The Higgs production mechanism considered here is the gluon-gluon fusion, with the assumed cross-section $\sigma=48.61 \mathrm{pb}_{-3.15 \mathrm{pb}}^{+2.08 \mathrm{pb}}$ (theory) $\pm 0.89 \mathrm{pb}(\mathrm{PDF})_{-1.26 \mathrm{pb}}^{+1.24 \mathrm{pb}}\left(\alpha_{s}\right)$, as reported in Ref. [12]. In the FRVZ model the scenario with the decay chain initiated by a heavier Higgs-like scalar $H^{\prime}\left(m_{H^{\prime}}=800 \mathrm{GeV}\right)$ is studied too.

\section{Mono-jet search}

A monojet topology is obtained when an energetic jet recoils against large $E_{\mathrm{T}}^{\text {miss }}$. The monojet Signal Region (SR), as in Ref. [1], is thus defined requiring $E_{\mathrm{T}}^{\mathrm{miss}}>200 \mathrm{GeV}$ and a leading jet with $p_{\mathrm{T}}>150 \mathrm{GeV}$ and $|\eta|<2.4$. As no other particles should be detected, a lepton and $\gamma$ veto is added too. Thus the dominant backgrounds are the QCD multijets with badly reconstructed $E_{\mathrm{T}}^{\text {miss }}$, the noncollisional backgrounds such as cosmic rays and beam induced backgrounds, and the V+Jets, with 
the $Z(v v)$ being irreducible. The QCD background is suppressed by requiring up to four jets with $p_{\mathrm{T}}>30 \mathrm{GeV}$ and $|\eta|<2.8$ and an angular separation between the missing transverse momentum direction and any jet $\Delta_{\phi}^{\min }>0.4(0.6)$ for $E_{\mathrm{T}}^{\mathrm{miss}}>(\leq) 250 \mathrm{GeV}$. The residual contributions of QCD and non-collisional backgrounds are estimated through pure data-driven techniques. To constrain the normalization of the other SM backgrounds, Control Regions (CRs), each enriched by a specific background, are used. A simultaneous shape fit exploiting the distribution of the total transverse momentum recoiling against hadronic activity in the events $\left(p_{\mathrm{T}}^{\text {recoil }}\right)$ is performed. The $p_{\mathrm{T}}^{\text {recoil }}$ distributions for the SM background and the three benchmark scenarios introduced in Section 1 are shown in Figure 2. The fit of the $p_{\mathrm{T}}^{\text {recoil }}$ distributions is performed firstly on CRs only, to assess

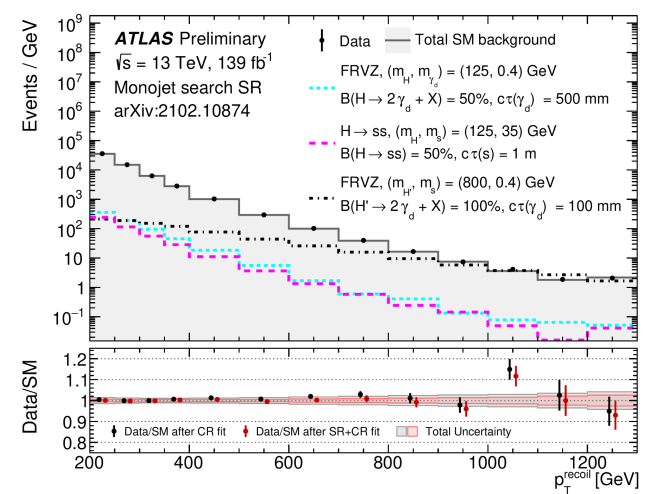

Figure 2: SR $p_{\mathrm{T}}^{\text {recoil }}$ distributions for the residual background and the studied signal models, as in [4]. The bottom pad shows the agreement between the data and the SM prediction, with black and red points indicating respectively the result after a fit to the CRs and after a fit to CRs + SR in the background-only hypothesis.

the agreement between SM predictions and data in SR, and then simultaneously on CRs and SR, to set limits on the signal strengths of the new physics models considered. As shown in Figure 2, no excess of data over SM predictions is observed after a fit to the CRs only, and the result of a simultaneous fit to the CRs and the SR, under the background-only hypothesis, shows how nominal SM predictions are only slightly affected when including the SR in the fit. Thus limits on the signal strengths of the BSM scenarios under study are set, providing new excluded areas in the parameters space of these models.

\section{Results and conclusions}

Upper limits at $95 \%$ of $\mathrm{CL}$ on $\mathcal{B}\left(H \rightarrow 2 \gamma_{d}+X\right)$ and on $\mathcal{B}\left(H^{\prime} \rightarrow 2 \gamma_{d}+X\right)$ for a $\gamma_{d}$ with $m_{\gamma_{d}}=400 \mathrm{MeV}$ are reported, in Figure 3(a) and Figure 3(b) respectively, as a function of $c \tau_{d}$ for both this work [4] and the dedicated displaced search [8].

Three separate channels are considered: the muonic channel (both $\gamma_{d} \rightarrow \mu \mu$ ), the mixed channel (one $\gamma_{d} \rightarrow \mu \mu$ and one $\gamma_{d} \rightarrow e e(q \bar{q})$ ) and the hadronic channel (both $\gamma_{d} \rightarrow e e(q \bar{q})$ ). At small $c \tau_{d}$ the analysis efficiency varies for the different $\gamma_{d}$ decay modes since the $\gamma_{d}$ decay products have to meet different criteria to be reconstructed, while at large $c \tau_{d}$, where competitive constraints are set, the sensitivity of this reinterpretation is similar in all the channels and results in complementary sensitivities with respect to the dedicated searches. The results of this work are 


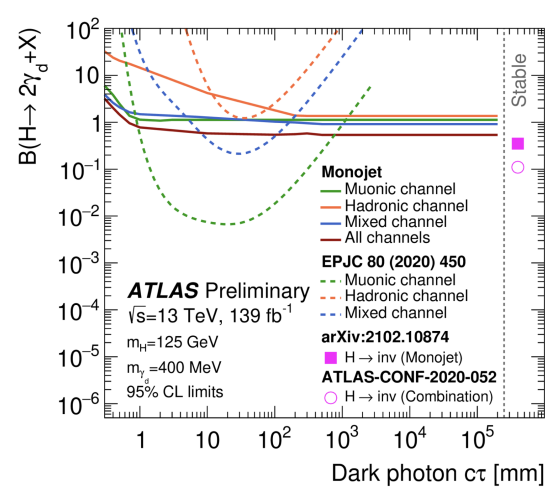

(a)

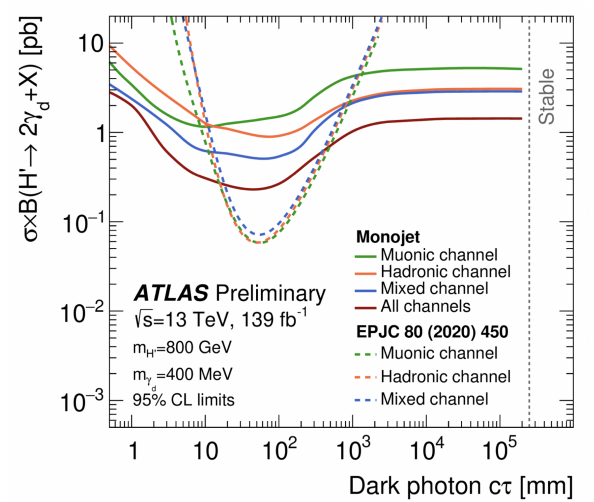

(b)

Figure 3: Upper limits on $\mathcal{B}\left(H \rightarrow 2 \gamma_{d}+X\right)$ and on $\mathcal{B}\left(H^{\prime} \rightarrow 2 \gamma_{d}+X\right)$ (Figure (a) and (b) respectively) for a $\gamma_{d}$ with $m_{\gamma_{d}}=400 \mathrm{MeV}$ in the muonic (green), hadronic (orange) and mixed (blue) channels. The red line is the exclusion limit obtained in this study [4] combining the three channels. The results are compared to the ones of the dedicated ATLAS search (dashed lines with same colour scheme) [8]. The $\mathcal{B}(H \rightarrow$ inv) limit from the monojet [1] analysis and the combination of ATLAS Run-1 and Run-2 results [13] are also shown.

shown at $90 \%$ of $\mathrm{CL}$ too in the $\left(\varepsilon, m_{\gamma_{d}}\right)$ plane for a fixed $\mathcal{B}\left(H \rightarrow 2 \gamma_{d}+X\right)=50 \%$ in Figure 4(a), together with the results of the prompt [7] and displaced [8] ATLAS analyses.

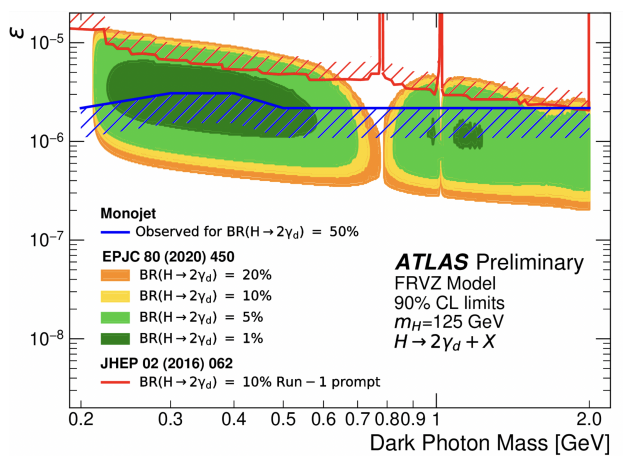

(a)

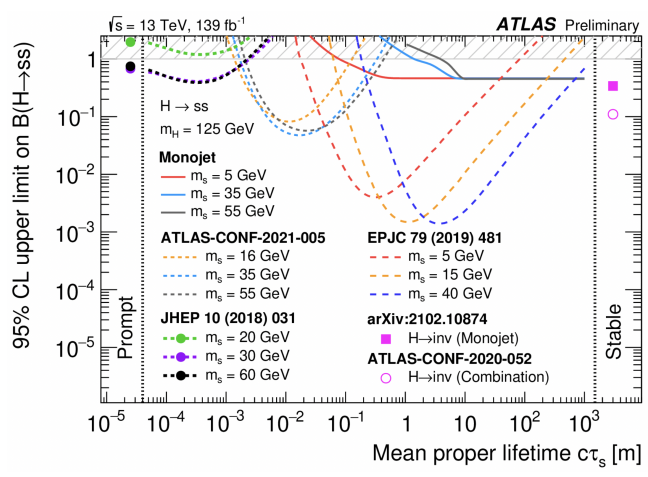

(b)

Figure 4: In Figure (a) the $90 \%$ CL exclusion contours in the $\left(\varepsilon, m_{\gamma_{d}}\right)$ plane obtained in this reinterpretation [4] are compared to the results of the dedicated prompt [7] and displaced [8] ATLAS searches. In Figure (b) 95\% CL exclusion contours on $\mathcal{B}(H \rightarrow s s)$ obtained in this study [4] are shown as a function of $c \tau_{s}$ and are compared to the ones of dedicated searches [9, 10, 11]. The $\mathcal{B}(H \rightarrow$ inv $)$ limit from the monojet [1] search and the combination of ATLAS Run-1 and Run-2 results [13] are shown too.

Upper limits at $95 \%$ of $\mathrm{CL}$ on $\mathcal{B}(H \rightarrow s s)$ are reported in Figure $4(\mathrm{~b})$ as a function of $c \tau_{s}$ for both this work and other dedicated searches. The complementarity among the several analyses can be observed for all $m_{s}$, for which different $c \tau_{s}$ are probed by different detection techniques, and the monojet reinterpretation is able to extend the current upper limits to larger $c \tau_{s}$.

It's worth noting that for the models with the SM Higgs (Figures 3(a) and 4(b)) at large $c \tau$ this study is less sensitive than Ref.[1]; this is due to the fact that here only $g g \rightarrow H$ is considered as Higgs production mechanism, while in [1] all Higgs production mechanisms are taken into account. 


\section{References}

[1] ATLAS Collaboration. "Search for new phenomena in events with an energetic jet and missing transverse momentum in $p p$ collisions at $\sqrt{s}=13 \mathrm{TeV}$ with the ATLAS detector". In: Phys. Rev. D 103.11 (2021), p. 112006. DoI: 10.1103/PhysRevD. 103.112006. arXiv: 2102.10874 [hep-ex].

[2] ATLAS Collaboration. "The ATLAS Experiment at the CERN Large Hadron Collider". In: JINST 3 (2008), S08003. DOI: 10 . 1088/1748-0221/3/08/S08003.

[3] Lyndon Evans and Philip Bryant. "LHC Machine". In: JINST 3 (2008), S08001. DoI: 10. 1088/1748-0221/3/08/S08001.

[4] ATLAS Collaboration. Constraining the Dark Sector with the monojet signature in the ATLAS experiment. ATL-PHYS-PUB-2021-020. URL: http: //cds . cern.ch/record/2772627? ln=it.

[5] Adam Falkowski et al. "Hidden Higgs Decaying to Lepton Jets". In: JHEP 05 (2010), p. 077. DOI: $10.1007 /$ JHEP05 (2010)077. arXiv: 1002.2952 [hep-ph].

[6] Adam Falkowski et al. "Discovering Higgs Decays to Lepton Jets at Hadron Colliders". In: Phys. Rev. Lett. 105 (2010), p. 241801. Dor: 10.1103/PhysRevLett. 105 . 241801. arXiv: 1007.3496 [hep-ph].

[7] ATLAS Collaboration. "A search for prompt lepton-jets in $p p$ collisions at $\sqrt{s}=8 \mathrm{TeV}$ with the ATLAS detector". In: JHEP 02 (2016), p. 062. DOI: 10.1007 / JHEP02 (2016) 062. arXiv: 1511.05542 [hep-ex].

[8] ATLAS Collaboration. "Search for light long-lived neutral particles produced in $p p$ collisions at $\sqrt{s}=13 \mathrm{TeV}$ and decaying into collimated leptons or light hadrons with the ATLAS detector". In: Eur. Phys. J. C 80 (2020), p. 450. DoI: 10.1140/epjc/s10052-020-79974. arXiv: 1909.01246 [hep-ex].

[9] ATLAS Collaboration. "Search for the Higgs boson produced in association with a vector boson and decaying into two spin-zero particles in the $H \rightarrow a a \rightarrow 4 b$ channel in $p p$ collisions at $\sqrt{s}=13 \mathrm{TeV}$ with the ATLAS detector". In: JHEP 10 (2018), p. 031. DOI: 10.1007/JHEP10 (2018)031. arXiv: 1806.07355 [hep-ex].

[10] ATLAS Collaboration. "Search for long-lived neutral particles in $p p$ collisions at $\sqrt{s}=13$ $\mathrm{TeV}$ that decay into displaced hadronic jets in the ATLAS calorimeter". In: Eur. Phys. J. C 79.6 (2019), p. 481. DoI: 10.1140 /epjc/s10052-019-6962-6. arXiv: 1902.03094 [hep-ex].

[11] ATLAS Collaboration. Search for exotic decays of the Higgs boson to long-lived particles in pp collisions at $\sqrt{s}=13 \mathrm{TeV}$ using displaced vertices in the ATLAS inner detector. ATLAS-CONF-2021-005. 2021. URL: https: //cds . cern.ch/record/2759209.

[12] M. Cepeda et al. "Report from Working Group 2: Higgs Physics at the HL-LHC and HELHC". In: CERN Yellow Rep. Monogr. 7 (2019). Ed. by Andrea Dainese et al., pp. 221-584. DOI: 10.23731/CYRM-2019-007.221. arXiv: 1902.00134 [hep-ph]. 
[13] ATLAS Collaboration. Combination of searches for invisible Higgs boson decays with the ATLAS experiment. ATLAS-CONF-2020-052. 2020. URL: https : //cds . cern . ch/record/ 2743055. 